\title{
Index of Sources
}

\begin{tabular}{|c|c|c|c|}
\hline \multicolumn{2}{|c|}{ Hebrew Bible } & $23: 35$ & 386 \\
\hline \multicolumn{2}{|l|}{ Genesis } & $23: 39-45$ & 387 \\
\hline 1 & 216,412 & $24: 1-4$ & 142 \\
\hline 22 & 24 & & \\
\hline 24 & 24 & Numbers & \\
\hline $35: 22 b-26$ & $164,166-167$ & 1 & 105 \\
\hline $46: 11$ & 64 & 2 & 163 \\
\hline \multirow[t]{2}{*}{49} & 166 & $3-4$ & 64,66 \\
\hline & & $3: 14-39$ & 70 \\
\hline \multicolumn{2}{|l|}{ Exodus } & 4 & 18 \\
\hline $1: 2-4$ & 164 & $4: 3$ & 303 \\
\hline $4: 10-17$ & 72 & $4: 15,19 b$ & 77 \\
\hline $6: 14-25$ & 314,318 & $6: 1-2$ & 93 \\
\hline $6: 16$ & $62-64$ & $9-10$ & 64 \\
\hline $6: 23$ & 65 & $9: 1-14$ & $232-233,234,236-237$ \\
\hline $6: 25$ & $67-68$ & $9: 4-5$ & 115 \\
\hline $24: 1,9-11$ & 66 & 10 & $79-83$ \\
\hline 15 & 71 & 11 & 87 \\
\hline 23 & 383 & 12 & $268-269,275$ \\
\hline 28 & $135-146$ & 16 & $73,135,275-276,136$ \\
\hline $28: 36-38$ & 270 & $17: 1-5$ & 67 \\
\hline $29: 38-42$ & 322 & $17: 6-15$ & 156 \\
\hline $29: 57$ & 271 & 18 & $85,94,341,352-354$ \\
\hline \multicolumn{2}{|l|}{ 30:1-10 } & & $411-412$ \\
\hline $30: 7-8$ & $130,142,226$ & $20-28$ & 75 \\
\hline $32: 29$ & 252 & $20: 19$ & 111 \\
\hline $37-40$ & 43,49 & $25: 8$ & 70 \\
\hline 39:30 & 271 & $25: 12-13$ & 69 \\
\hline \multirow[t]{2}{*}{$40: 17$} & $228,232-233$ & 26 & $66,84,96,103,105$ \\
\hline & & 27 & $101-102,156$ \\
\hline \multicolumn{2}{|l|}{ Leviticus } & 28 & 178 \\
\hline $1-7$ & $175-179$ & $28-29$ & 240 \\
\hline $8-10$ & $233-242$ & 29 & 383 \\
\hline 8:9 & 271 & 30 & $89-90$ \\
\hline 10 & $61-62,66,134-135,275$ & 31 & $88-89,94,103,112,113$ \\
\hline $10: 2$ & 73 & $31: 19$ & 111 \\
\hline $12-15$ & $185,188-189,190$ & 32 & 111 \\
\hline $13-14$ & 269 & & \\
\hline \multirow[t]{2}{*}{16} & $135,156,182-184,221$, & Deuteronomy & \\
\hline & $226,228-231$ & $7: 5$ & 362 \\
\hline $17-26$ & $190-191$ & 9:5 & 342 \\
\hline $17: 7$ & 365 & $10: 8$ & 258 \\
\hline 18 & 188 & 12 & 56 \\
\hline 23 & 383 & 16 & $232,240,383$ \\
\hline
\end{tabular}




\begin{tabular}{|c|c|c|c|}
\hline 17 & 58 & 2 Kings & \\
\hline $18: 6-7$ & 367 & 5 & 268 \\
\hline $18: 15-22$ & 72 & $11-12$ & $142-148$ \\
\hline 20:13-14 & $94-95$ & $12: 10$ & 139 \\
\hline $23: 4-7$ & 382 & $12: 21-22$ & 87 \\
\hline 23:22 & 93 & 15 & 275,361 \\
\hline $29: 16$ & 362 & $15: 2$ & 264 \\
\hline $31: 9,25$ & 258 & $15: 33$ & 343 \\
\hline 32:39 & $71-72$ & $18: 4$ & 179 \\
\hline $34: 7$ & 148 & $22: 4$ & 139 \\
\hline \multirow[t]{2}{*}{$34: 10-12$} & 59 & 23 & $361,366-369$ \\
\hline & & $23: 4$ & 153 \\
\hline \multicolumn{4}{|l|}{ Joshua } \\
\hline 6 & 88 & Isiah & \\
\hline $13-22$ & $111-112,120$ & $37: 2$ & 203 \\
\hline 17 & 97 & 56 & 56 \\
\hline 20 & 97 & $66: 21$ & 285 \\
\hline 21 & $64,71,121,364$ & & \\
\hline \multirow[t]{2}{*}{22} & 70 & Jeremiah & \\
\hline & & $1: 11-12$ & 74 \\
\hline Judges & & $9: 21-10: 22$ & 213 \\
\hline 11 & 176 & $23: 5$ & 274 \\
\hline \multirow[t]{2}{*}{$20: 28$} & 68,70 & $33: 5$ & 274 \\
\hline & & $33: 14-22$ & 285 \\
\hline 1 Samuel & & 36 & 203 \\
\hline 8 & 58 & & \\
\hline 13 & 176,178 & Ezekiel & \\
\hline \multirow[t]{2}{*}{27} & 116 & $1: 26-28$ & 66 \\
\hline & & $10: 1-2$ & 66 \\
\hline 2 Samuel & & $17: 11-17$ & 357 \\
\hline 6 & 82 & $27: 13$ & 108 \\
\hline $6: 5$ & 81 & 34 & 69 \\
\hline $6: 12-19$ & 284 & $40: 45-46$ & 366 \\
\hline 7 & 246 & $42: 12-13$ & 337,339 \\
\hline \multirow[t]{2}{*}{$24: 9$} & 104 & 43 & 241 \\
\hline & & $43-45$ & $234-238$ \\
\hline 1 Kings & & 43:11 & 329 \\
\hline $1-2$ & 330 & $43: 24$ & 85 \\
\hline 2 & 62 & 44 & $60-61,70,73,77$ \\
\hline $4: 2$ & 344 & & $340-341,349-356,359$ \\
\hline $6-8$ & 329,335 & & $362,370-374,391$ \\
\hline 7 & 105 & $44-45$ & 345 \\
\hline 8 & $77,226,239$ & $44: 10-12$ & 344 \\
\hline $9: 25$ & 240 & $47-48$ & $159-163,169-172$ \\
\hline $11: 14 ; 12: 14$ & 80,83 & $48: 11$ & 70 \\
\hline
\end{tabular}




\begin{tabular}{|c|c|c|c|}
\hline Hosea & & $10: 29$ & 398,415 \\
\hline \multirow[t]{2}{*}{$5: 8$} & 80 & 11 & 398,407 \\
\hline & & 12 & $81,387-388,399,407$ \\
\hline Amos & & 13 & $380-382,388,409$ \\
\hline \multirow[t]{2}{*}{525} & 73 & & \\
\hline & & 1 Chronicles & \\
\hline Haggai & & $1-9$ & $163-167$ \\
\hline \multirow{2}{*}{$1: 1$} & 55 & 5 & $112,115-117,121$ \\
\hline & & $5-6$ & $250,312-316$ \\
\hline Zechariah & & $5: 18-22$ & $103,109,114$ \\
\hline $12: 12-14$ & 286 & $5: 27-41$ & $62,64,68,70,127$ \\
\hline \multirow[t]{2}{*}{14} & 286 & $6: 16-17$ & 76 \\
\hline & & $6: 34$ & 129-131, 176 \\
\hline Malachi & & $6: 35-38$ & 63,71 \\
\hline \multirow[t]{2}{*}{$2: 1-9 ; 31-4$} & 287 & 7 & 103 \\
\hline & & 9 & 84,293 \\
\hline$J o b$ & & 15 & $253-254,293$ \\
\hline $32: 13$ & $93,97-98$ & 16 & $39-40,293$ \\
\hline \multirow[t]{2}{*}{$32: 13-14$} & 98 & $21: 5-6$ & 104 \\
\hline & & $23: 24-27$ & 303 \\
\hline Daniel & & $23: 28-32$ & $177,358-359$ \\
\hline 4 & 214 & $23: 29$ & 177 \\
\hline \multirow[t]{2}{*}{7} & 214 & $23-27$ & 331 \\
\hline & & $25: 4$ & 86 \\
\hline Ezra & & $25: 5$ & 93,95 \\
\hline 1 & $381-382$ & 28 & $324-329,334$ \\
\hline $1: 5-8$ & 55,379 & $28: 18$ & 327 \\
\hline 2 & 158,414 & $28: 19$ & $327-328$ \\
\hline $2: 43-54$ & 354 & 29 & 106,330 \\
\hline $2: 62-63$ & 310,313 & & \\
\hline 3 & $382-384$ & 2 Chronicles & \\
\hline 6 & 395,413 & $2: 3$ & 383 \\
\hline $6: 20$ & $399,406,415$ & $5-8$ & $239-242$ \\
\hline 7 & 70 & 813 & 383 \\
\hline $7-8$ & $384-386,395$ & $11: 1$ & 245 \\
\hline $7: 1-6$ & 202 & 11:13-17 & $363-365$ \\
\hline $7: 5$ & 68,70 & 13 & 136 \\
\hline $9: 1-2$ & $396-398,412$ & $13: 5$ & 85 \\
\hline \multirow[t]{2}{*}{$10: 16$} & 396 & 14 & $115-117$ \\
\hline & & 16 & 134 \\
\hline Nehemiah & & 19:11 & $137,138-139$ \\
\hline \multirow[t]{2}{*}{7} & $158,307,313,406,408$ & 20 & 96 \\
\hline & 411 & $20: 13$ & 89,92 \\
\hline $7: 7$ & 55 & $20: 28$ & 80,82 \\
\hline 8 & 395 & $22: 11$ & 143 \\
\hline $8-9$ & $386-387$ & $23: 3$ & 144 \\
\hline 10 & 409 & 23:9-10 & 145 \\
\hline
\end{tabular}




$\begin{array}{ll}23: 11 & 146 \\ 23: 16 & 147 \\ 23: 18-19 & 138 \\ 24: 15-16(18) & 66,148 \\ 25 & 115-117 \\ 26: 16-21 & 132,135,141,238-239 \\ & 260-279 \\ 29 & 192-193,337-340 \\ & 341-342,344 \\ 29-31 & 150-154,179-180 \\ & 182-186,234-237 \\ 29: 34 & 301 \\ 30 & 233 \\ 30: 17-19 & 190 \\ 30: 18 & 187 \\ 31: 10 & 138,342-343 \\ 34: 3-5 & 188\end{array}$

\section{New Testament}

Luke

5:39

49

\section{Qumran Texts}

1QS 2.19-23 295

1QS 6.3-9 292

1QS 7.12-21 215

1QWar 7.9-18 292

4Q71 213

4Q242 214

4Q 267-273 215

$\begin{array}{ll}4 Q D^{\mathrm{a}-\mathrm{g}} & 215 \\ \text { CD 8-9 } & 215 \\ \text { CD 14.3-6 } & 292 \\ \text { CD 15-16 } & 215 \\ \text { CD 19-20 } & 215\end{array}$

\section{Elephantine}

TAD A3.3:5 204

TAD A4.1-10 204

TAD A6.1:1, $6 \quad 204$

TAD A6.2:28 204

TAD B4.3:13 204

\section{Josephus}

Ant $6.15 \quad 284$

Ant $10.331 \quad 274$

Ant 11.297-301 140

Ant 20.216-8 292, 293

Apio II 164-5 53-54

Apio II 184-7 54

\section{Philo}

Spec 1.156-58 291, 293

Spec $1.79 \quad 291$

Spec 3.126-7 291

Sacr 118, 127-30 291

Sacr 119-120 292

Moses 2.174-9 291

Moses 2.276-87 291

Worse $135 \quad 292$ 